\title{
Effective metastability for modified Halpern iterations in CAT(0) spaces
}

\section{K Schade and U Kohlenbach*}

\section{"Correspondence:}

kohlenbach@mathematik.

tu-darmstadt.de

Department of Mathematics,

Technische Universität Darmstadt, Schlossgartenstrasse 7, Darmstadt, 64289, Germany

\begin{abstract}
We examine convergence results for modified Halpern iterations due to Cuntavepanit and Panyanak (Fixed Point Theory Appl. 2011:869458, 2011). Following Kohlenbach and Leuştean (Adv. Math. 231:2525-2556, 2012), we extract uniform rates of metastability. This includes extracting rates of asymptotic regularity and replacing an ineffective argument that uses Banach limits.
\end{abstract}

Keywords: modified Halpern iteration; metastability; rate of asymptotic regularity; proof mining; CAT(0) space

\section{Introduction}

Recently, Kohlenbach and Leuştean developed a method for analyzing convergence proofs that make use of Banach limits (and hence - for what is known - the axiom of choice) and applied this method to obtain quantitative versions of convergence results for Halpern iterations in $\mathrm{CAT}(0)$ and uniformly smooth spaces (see $[1,2])$. In this paper we apply this method to a recent convergence proof (again in the CAT(0)-setting) due to Cuntavepanit and Panyanak [3] for a modified scheme of Halpern iterations due to Kim and $\mathrm{Xu}$ [4].

Given a nonempty convex subset $C$ in a $\operatorname{CAT}(0)$ space, we consider $u, x \in C$, a sequence $\left(\lambda_{n}\right) \subset[0,1]$ and a nonexpansive mapping $T: C \rightarrow C$ with a nonempty fixed point set. Then the Halpern iterations with an initial point $x$ and a reference point $u$ are given by

$$
x_{0}:=x, \quad x_{n+1}:=\lambda_{n} u \oplus\left(1-\lambda_{n}\right) T x_{n}, \quad \text { for } n \in \mathbb{N} \text {. }
$$

Here, for $x, y \in X$ and $\lambda \in[0,1], \lambda x \oplus(1-\lambda) y$ denotes the unique point $z \in X$ with $d(z, x)=$ $(1-\lambda) d(x, y)$ and $d(z, y)=\lambda d(x, y)$.

A particularly important choice for $\lambda_{n}$ is $\left(\frac{1}{n+2}\right)_{n \in \mathbb{N}}$. Then if $T$ is linear and $u$ is chosen equal to $x$, one obtains the Cesàro averages of $\left(T^{n} x\right)_{n \in \mathbb{N}}$

$$
x_{n}=\frac{1}{n+1} \sum_{i=0}^{n} T^{i} x
$$

The conditions used in this paper always allow for this choice of $\left(\lambda_{n}\right)$.

In [1], Kohlenbach and Leuştean extracted both effective rates of convergence for the asymptotic regularity property

$$
d\left(x_{n}, T x_{n}\right) \rightarrow 0
$$

2012 Schade and Kohlenbach; licensee Springer. This is an Open Access article distributed under the terms of the Creative Commons Attribution License (http://creativecommons.org/licenses/by/2.0), which permits unrestricted use, distribution, and reproduction in any medium, provided the original work is properly cited. 
and effective so-called rates of metastability (in the sense of Tao [5]) for the convergence of the sequence $\left(x_{n}\right)$ of Halpern iterations applying techniques of the proof mining program (see [6] for general information) to a convergence proof due to Saejung [7]. Here, by a rate of metastability, we mean a function $\Psi:(0, \infty) \times \mathbb{N}^{\mathbb{N}} \rightarrow \mathbb{N}$ such that

$$
\forall \varepsilon>0, \forall g: \mathbb{N} \rightarrow \mathbb{N}, \exists N \leq \Psi(\varepsilon, g), \forall m, n \in[N ; N+g(N)] \quad\left(d\left(x_{n}, x_{m}\right) \leq \varepsilon\right),
$$

where $[N ; N+m]:=\{N, N+1, N+2, \ldots, N+m]$.

In general, there is no computable rate of convergence for Halpern iterations $\left(x_{n}\right)_{n \in \mathbb{N}}$ (already for $\lambda_{n}:=1 /(n+2)$ and linear $\left.T\right)$ as follows from [8, Theorem 5.1]. Note, however, that the metastability property

$$
\forall \varepsilon>0, \forall g: \mathbb{N} \rightarrow \mathbb{N}, \exists N \in \mathbb{N}, \forall m, n \in[N ; N+g(N)] \quad\left(d\left(x_{n}, x_{m}\right) \leq \varepsilon\right)
$$

(for which [1] does extract effective rates) ineffectively is equivalent to the usual Cauchy property.

Saejung's original proof makes substantial reference to the axiom of choice by using the existence of Banach limits. Kohlenbach and Leuștean eliminated this reference in favor of the use of a finitary functional which renders Saejung's proof admissible for the proof mining program. In this paper we apply this method to a variation of Halpern iterations, the aforementioned modified Halpern iterations due to [4].

In the same setting as above, we consider two sequences $\left(\alpha_{n}\right)_{n \in \mathbb{N}},\left(\beta_{n}\right)_{n \in \mathbb{N}} \subset[0,1]$. Then the modified Halpern iterations with an initial point $x$ and a reference point $u$ are given by

$$
x_{0}:=x, \quad x_{n+1}:=\beta_{n} u \oplus\left(1-\beta_{n}\right)\left(\alpha_{n} x_{n} \oplus\left(1-\alpha_{n}\right) T x_{n}\right), \quad \text { for } n \in \mathbb{N} .
$$

So, instead of using $T x_{n}$ as in the usual Halpern iteration, one takes here the so-called Krasnoselski-Mann iteration

$$
x_{0}:=x, \quad x_{n+1}:=\alpha_{n} x_{n} \oplus\left(1-\alpha_{n}\right) T x_{n} .
$$

Modified Halpern iterations can be seen as generalizations of Halpern iterations by putting $\alpha_{n} \equiv 0$, and so our results (which allow this choice) extend the quantitative metastability and asymptotic regularity results of [1]. The main convergence result that we treat is due to Cuntavepanit and Panyanak [3]. Even for ordinary Halpern iterations, the inclusion of the case of unbounded $C$ in our bounds is new compared to [1]. Our paper further strengthens the claim made in [1] to have developed a general method for analyzing quantitatively strong convergence proofs that use Banach limits.

\section{Preliminaries}

CAT(0) spaces are instances of geodesic spaces which are special metric spaces. Roughly speaking, in a geodesic space the associated metric behaves in an orderly manner, i.e., making sure there is at least one shortest path between two points. A CAT(0) space enforces further regularity in the sense that every triangle in the space is as 'thin' as in the Euclidean space.

The terminology of CAT $(\kappa)$ spaces is due to Gromov [9]. CAT(0) spaces are uniquely geodesic [10, Proposition II.1.4(1)]. 
By $(1-\lambda) x \oplus \lambda y$ we denote the unique point $z$ on the unique geodesic segment $[x, y]$ joining $x$ and $y$ so that

$$
d(z, x)=\lambda d(x, y) \quad \text { and } \quad d(z, y)=(1-\lambda) d(x, y)
$$

holds.

The following properties of CAT(0) spaces are of interest to us.

Proposition 2.1 ([11, Lemma 2.5]) Let $X$ be a CAT(0) space. Then the following inequality holds for all $x, y, z \in X$ and for all $t \in[0,1]$ :

$$
d((1-t) x \oplus t y, z)^{2} \leq(1-t) d(x, z)^{2}+t d(y, z)^{2}-t(1-t) d(x, y)^{2} .
$$

For a uniquely geodesic space $X$, this property is equivalent to $X$ being a CAT(0) space.

Proposition 2.2 ([11, Lemma 2.4]) Let $X$ be a CAT(0) space. If $x, y, z$ are points in $X$ and $t \in[0,1]$, then

$$
d((1-t) x \oplus t y, z) \leq(1-t) d(x, z)+t d(y, z),
$$

i.e., CAT(0) spaces are, in particular, convex metric spaces in the sense of Takahashi [12] by taking $W(x, y, \lambda):=(1-\lambda) x \oplus \lambda y$.

Every pre-Hilbert space is a CAT(0)-space. Another example is the open unit ball $B$ in $\mathbb{C}$ with the Poincaré metric,

$$
\rho(z, w):=2 \tanh ^{-1}\left|\frac{z-w}{1-z \bar{w}}\right| \quad \text { for } z, w \in B
$$

This example is interesting for fixed point theory since holomorphic mappings $f: B \rightarrow B$ are nonexpansive with respect to $\rho$ (Schwarz-Pick lemma, see [13]). $\mathbb{R}$-trees in the sense of Tits are further examples of CAT( 0$)$ spaces.

W-hyperbolic spaces are in turn generalizations of $\operatorname{CAT}(0)$ spaces. The following definition of W-hyperbolic spaces is due to Kohlenbach [14, Definition 2.11].

Definition 2.3 A triple $(X, d, W)$ is called a W-hyperbolic space if $(X, d)$ is a metric space and $W: X \times X \times[0,1] \rightarrow X$ is a mapping satisfying

(W1) $d(z, W(x, y, \lambda)) \leq(1-\lambda) d(z, x)+\lambda d(z, y)$,

(W2) $d\left(W\left(x, y, \lambda_{0}\right), W\left(x, y, \lambda_{1}\right)\right)=\left|\lambda_{0}-\lambda_{1}\right| d(x, y)$,

(W3) $W(x, y, \lambda)=W(y, x, 1-\lambda)$,

(W4) $d(W(x, z, \lambda), W(y, w, \lambda)) \leq(1-\lambda) d(x, y)+\lambda d(z, w)$.

Lemma 2.4 $([6, \mathrm{p} .386])$ Let $(X, d)$ be a $C A T(0)$ space. If it is equipped with the mapping $W: X \times X \times[0,1] \rightarrow X$,

$$
W(x, y, \lambda):=(1-\lambda) x \oplus \lambda y,
$$

W satisfies (W1)-(W4), i.e., $(X, d, W)$ is W-hyperbolic. 
We will use the following notions (here and in the following, $\mathbb{N}$ is the set of natural numbers including 0 , while $\mathbb{Z}_{+}$denotes the set of natural numbers $n \geq 1$ ):

(1) A mapping $\gamma:(0, \infty) \rightarrow \mathbb{Z}_{+}$is called a Cauchy modulus of a Cauchy sequence $\left(a_{n}\right)_{n \in \mathbb{N}}$ in a metric space $(X, d)$ if

$$
\forall \varepsilon>0, \forall n \in \mathbb{N} \quad\left(d\left(a_{\gamma(\varepsilon)+n}, a_{\gamma(\varepsilon)}\right) \leq \varepsilon\right) .
$$

(2) For $\left(a_{n}\right)_{n \in \mathbb{N}}$ as above, a mapping $\Psi:(0, \infty) \times \mathbb{N}^{\mathbb{N}} \rightarrow \mathbb{Z}_{+}$is a rate of metastability if

$$
\forall \varepsilon>0, \forall g: \mathbb{N} \rightarrow \mathbb{N}, \exists N \leq \Psi(\varepsilon, g), \forall m, n \in[N ; N+g(N)] \quad\left(d\left(a_{n}, a_{m}\right) \leq \varepsilon\right) .
$$

(3) Let $\left(a_{n}\right)_{n \in \mathbb{N}}$ be a sequence in $\mathbb{R}$. If $\lim _{\sup } \rightarrow \infty a_{n} \leq 0$, then a mapping $\theta:(0, \infty) \rightarrow \mathbb{Z}_{+}$is called an effective rate for $\left(a_{n}\right)_{n \in \mathbb{N}}$ if

$$
\forall \varepsilon>0, \forall n \geq \theta(\varepsilon) \quad\left(a_{n} \leq \varepsilon\right) .
$$

(4) Let $\left(a_{n}\right)_{n \in \mathbb{N}}$ be a sequence of nonnegative reals such that $\sum_{n=1}^{\infty} a_{n}=\infty$. Then a function $\delta: \mathbb{Z}_{+} \rightarrow \mathbb{Z}_{+}$with

$$
\sum_{i=1}^{\delta(n)} a_{i} \geq n \quad \text { for all } n \in \mathbb{Z}_{+}
$$

is called a rate of divergence of $\left(\sum_{i=1}^{n} a_{i}\right)_{n \in \mathbb{N}}$.

The term metastability is due to Tao $[5,15]$. It is an instance of the no-counterexample interpretation by Kreisel [16, 17].

\section{Halpern iterations}

Definition 3.1 Let $C$ be a nonempty convex subset of a CAT(0) space X. Let $u, x \in C$, $\left(\alpha_{n}\right)_{n \in \mathbb{N}},\left(\beta_{n}\right)_{n \in \mathbb{N}} \subset[0,1]$ and $T: C \rightarrow C$ be nonexpansive. The modified Halpern iterations $x_{n} \in C$ with an initial point $x$ and a reference point $u$ are

$$
x_{0}:=x, \quad x_{n+1}:=\beta_{n} u \oplus\left(1-\beta_{n}\right)\left(\alpha_{n} x_{n} \oplus\left(1-\alpha_{n}\right) T x_{n}\right), \quad \text { for } n \in \mathbb{N} .
$$

Combinations of the following conditions were considered for the sequences $\left(\alpha_{n}\right)_{n \in \mathbb{N}}$ and $\left(\beta_{n}\right)_{n \in \mathbb{N}}$.

(D1)
(a) $\lim _{n \rightarrow \infty} \beta_{n}=0$,
(b) $\lim _{n \rightarrow \infty} \alpha_{n}=0$,
(a) $\sum_{n=0}^{\infty} \beta_{n}=\infty$
(b) $\sum_{n=0}^{\infty} \alpha_{n}=\infty$,

(D3)
(a) $\sum_{n=0}^{\infty}\left|\beta_{n}-\beta_{n+1}\right|<\infty$,
(b) $\sum_{n=0}^{\infty}\left|\alpha_{n}-\alpha_{n+1}\right|<\infty$,

(D4)
(a) $\prod_{n=0}^{\infty}\left(1-\beta_{n}\right)=0$,
(b) $\prod_{n=0}^{\infty}\left(1-\alpha_{n}\right)=0$,

(D5)

$$
0<\liminf _{n \rightarrow \infty} \beta_{n} \leq \limsup _{n \rightarrow \infty} \beta_{n}<1 .
$$

We will be concerned with (D1) to (D4). 
Modified Halpern iterations are a generalization of Halpern iterations if one is permitted to set $\alpha_{n}:=0$ for all $n \in \mathbb{N}$. This excludes (D2.b) which, however, we will never need.

Halpern iterations were named after a paper by Halpern [18] in 1967. This is somewhat misleading since Halpern considered only an instance of Halpern iterations in which the reference point was set to 0 and hence required a closed ball around 0 to be contained in the domain $C$ of the self-mapping $T$. In the paper, Halpern examined these iterations in the setting of Hilbert spaces. For the convergence of $\left(x_{n}\right)_{n \in \mathbb{N}}$ to a fixed point of $T$ with the smallest norm (hence closest to $u=0$ ), he showed that the conditions (D1.a) and (D2.a) were necessary. He also gave a set of sufficient conditions.

In 1977, Lions [19] improved Halpern's original result. He considered real Hilbert spaces and Halpern iterations in full generality in the article and showed the convergence of the iteration to the fixed point of $T$ nearest to $u$ under the following conditions: $\left(\lambda_{n}\right)_{n \in \mathbb{N}} \in$ $(0,1],(D 1 . a),(D 2 . a)$ and $\lim _{n \rightarrow \infty} \frac{\lambda_{n}-\lambda_{n+1}}{\lambda_{n+1}^{2}}=0$. Furthermore, he generalized his result to a variation of Halpern iterations which dealt with finite families of nonexpansive operators $T_{i}, 0 \leq i \leq N$, with $N \in \mathbb{N}$ instead of one nonexpansive $T$. Halpern and Lion's results did not cover the choice of $\left(\lambda_{n}\right)_{n \in \mathbb{N}}=\left(\frac{1}{n+2}\right)_{n \in \mathbb{N}}$.

In 1983, Reich [20] posed the following problem, which was referred to as Problem 6.

Let $X$ be a Banach space. Is there a sequence $\left(\lambda_{n}\right)_{n \in \mathbb{N}}$ such that whenever a weakly compact convex subset $C$ of $X$ possesses the fixed point property for nonexpansive mappings, then $\left(x_{n}\right)_{n \in \mathbb{N}}$ converges to a fixed point of $T$ for all $x \in C$ and all nonexpansive mappings $T: C \rightarrow C$ ?

Many partial answers have been given to this problem, we will only give a brief overview. The problem in its full generality is still open.

Wittmann [21] proved a result in 1992 which finally allowed for $\lambda_{n}=\frac{1}{n+2}$ in Hilbert spaces. While Halpern's proof relied on a limit theorem for a resolvent, Wittmann carried out a direct proof.

Theorem 3.2 (Wittmann [21, Theorem 2]) Let $C$ be a closed convex subset of a Hilbert space $X$ and $T: C \rightarrow C$ be a nonexpansive mapping with a fixed point. Assume $\left(\lambda_{n}\right)_{n \in \mathbb{N}}$ satisfies (D1.a), (D2.a) and (D3.a). Then for any $x \in C$, the Halpern iteration $\left(x_{n}\right)_{n \in \mathbb{N}}$ with $u=x \in C$ converges to the projection Px of $x$ on $\operatorname{Fix}(T)$.

The limit theorem on which Halpern's proof relied was generalized to uniformly smooth Banach spaces by Reich in [22]. In [23], Reich proved the strong convergence of $\left(x_{n}\right)$ in the setting of uniformly smooth Banach spaces that have a weakly sequentially continuous duality map for decreasing sequences of $\left(\lambda_{n}\right)_{n \in \mathbb{N}}$ satisfying (D1.a) and (D2.a).

In 1997, Shioji and Takahashi [24] considered Banach spaces with uniformly Gâteauxdifferentiable norm with a closed and convex subset $C$. They treated also the case $u \neq x$ for $u, x \in C$ for nonexpansive mappings $T: C \rightarrow C$ with a nonempty fixed point set and showed the convergence of $\left(x_{n}\right)_{n \in \mathbb{N}}$ to a fixed point if the conditions (D1.a), (D2.a) and (D3.a) held for $\left(\lambda_{n}\right)_{n \in \mathbb{N}} \subset[0,1]$ and for $0<t<1$, the sequence satisfying

$$
z_{t}:=t u+(1-t) T z_{t}
$$

converges strongly to $z \in \operatorname{Fix}(T)$ as $t \rightarrow 0$. The existence of this sequence follows from Banach's fixed point theorem. 
In 2010, Saejung [7] considered the case of complete CAT(0) spaces, which are a generalization of Hilbert spaces as already mentioned above and showed that for closed and convex subsets $C, T: C \rightarrow C$ nonexpansive with a nonempty fixed point set, and $\left(\lambda_{n}\right)_{n \in \mathbb{N}} \subset(0,1)$ satisfying the conditions (D1.a), (D2.a) to (D3.a) or, alternatively, (D1.a), (D2.a) and $\lim _{n \rightarrow \infty} \frac{\lambda_{n}}{\lambda_{n+1}}=1$, Halpern iterations converge strongly to the fixed point of $T$ nearest to $u$ (for the case of the Hilbert ball, which is a CAT(0) space, see already [13] and with further generalizations - [25]). Recently, Piạtek [26] has generalized - independently of Saejung's work - Saejung's result even to CAT $(\kappa)$-spaces with an argument which is new also for CAT(0) spaces and is more elementary than Saejung's approach based on Banach limits (it would be of interest to carry out a quantitative analysis of this proof due to Piątek). Saejung also studied Halpern iterations with finitely and countably many different nonexpansive mappings sharing a fixed point.

In 2011, Kohlenbach [27] considered Wittmann's proof and the case of $\lambda_{n}:=\frac{1}{n+2}$ for all $n \in \mathbb{N}$ for Halpern iterations in Hilbert spaces. He extracted a rate of metastability in both bounded and unbounded domains $C$. Subsequently, Kohlenbach and Leuștean [1] gave an effective uniform rate of metastability for Halpern iterations in CAT(0) spaces by analyzing Saejung's proof. They then treated arbitrary $\left(\lambda_{n}\right)_{n \in \mathbb{N}}$ satisfying (D1.a), either (D2.a) or (D4.a) and (D3.a) and the case of bounded C. As an intermediate step, they used (improved versions of) uniform effective rates of asymptotic regularity which were due to Leuştean [28] in 2007. In [2], Kohlenbach and Leuștean also develop a new metatheorem for real Banach spaces with a norm-to-norm uniformly continuous duality selection map. This metatheorem was then applied to the convergence proof of Halpern iterations by Shioji and Takahashi [24] for the extraction of rates of metastability in the setting of the metatheorem (though only relative to a given rate of metastability for the resolvent whose computation in this setting is still subject of ongoing research).

Kim and $\mathrm{Xu}$ [4] showed in 2005 the following result for their modified Halpern iteration from Definition 3.1:

Let $C$ be a closed convex subset of a uniformly smooth Banach space $X$, and let $T: C \rightarrow$ $C$ be a nonexpansive mapping with nonempty fixed point set. Under the conditions (D1)(D3) (a) + (b), $\left(x_{n}\right)_{n \in \mathbb{N}}$ converges strongly to a fixed point of $T$.

Independent of each other, Suzuki [29] in 2006 and Chidume and Chidume [30] in 2007 considered the following different iteration scheme:

$$
x_{0}:=x \in C, \quad y_{n+1}:=\alpha_{n} u \oplus+\left(1-\alpha_{n}\right)\left((1-\beta) x_{n} \oplus \beta T x_{n}\right) \quad \text { for } n \in \mathbb{N},
$$

for $\beta \in(0,1)$. By ruling out $\beta=1$, they excluded original Halpern iterations in their scheme.

Let $X$ be a Banach space with uniformly Gâteaux-differentiable norm, $C \subset X$ be a closed convex subset, $T: C \rightarrow C$ be nonexpansive with a nonempty fixed point set, $u, x \in C$. They showed convergence of this scheme to a fixed point of $T$ if $\left(\lambda_{n}\right)_{n \in \mathbb{N}} \subset[0,1]$ satisfies (D1.a) and (D2.a) and if $\left(z_{t}\right)_{t \in(0,1)}$ converges strongly to some point $z \in C$ as $t \rightarrow 0$, where $z_{t}$ is the unique element of $C$ with $z_{t}=t u+(1-t) T z_{t}$ for every $0<t<1$.

Note that Kim and Xu's result does not permit this iteration scheme since a constant $\beta \in(0,1)$ does not satisfy (D1.b).

In 2011, Cuntavepanit and Panyanak [3] generalized Kim and Xu's result to CAT(0) spaces and eliminated the use of condition (D2.b). They considered $C$ to be a nonempty closed convex subset of a complete CAT(0) space $X, x, u \in C$ and $T: C \rightarrow C$ to be a nonexpansive mapping with a nonempty fixed point set and showed strong convergence to 
the fixed point of $T$ nearest to $u$ of the modified Halpern iterations defined here under the conditions (D1.a), (D1.b), (D2.a), (D3.a) and (D3.b).

This scheme does not cover the schemes due to Suzuki and Chidume and Chidume, since the choice of $\left(\alpha_{n}\right)$ as $(1-\beta)$ is not permitted because of (D1.a). Since in [3] (D2.b) is no longer used, modified Halpern iterations can be viewed as generalizations of Halpern iterations.

Cuntavepanit and Panyanak also considered a different iteration scheme: For $\left(\alpha_{n}\right),\left(\beta_{n}\right) \subset$ $[0,1]$, let

$$
x_{0}:=x \in C, \quad x_{n+1}:=\beta_{n} x_{n} \oplus\left(1-\beta_{n}\right)\left(\alpha_{n} u \oplus\left(1-\alpha_{n}\right) T x_{n}\right), \quad \text { for } n \in \mathbb{N},
$$

and showed that the conditions (D1.b), (D2.b) and (D5) suffice for strong convergence in the above setting. We will call these iterations secondary modified Halpern iterations.

This scheme excludes original Halpern iterations. Setting for all $n \in \mathbb{N}$,

$$
\beta_{n}^{\prime}:=\left(1-\alpha_{n}\right)(1-\beta) \quad \text { and } \quad \alpha_{n}^{\prime}:=\alpha_{n} /\left(1-\beta_{n}^{\prime}\right)
$$

this scheme includes Chidume and Chidume's and Suzuki's iteration scheme, though. The quantitative analysis of the convergence proof for (5) has to be left for future research.

\section{Main results}

Theorem 4.1 Let $X$ be a complete $C A T(0)$ space, $C \subseteq X$ be a closed convex subset, $x, u \in C$ and $T: C \rightarrow C$ be nonexpansive with a nonempty fixed point set. Let $\left(\alpha_{n}\right)_{n \in \mathbb{N}},\left(\beta_{n}\right)_{n \in \mathbb{N}} \subset$ $[0,1]$ satisfy (D1.a) and (D1.b), (D2.a), (D3.a) and (D3.b). Then the modified Halpern iteration $\left(x_{n}\right)_{n \in \mathbb{N}}$ is Cauchy. Furthermore, let

$$
\begin{aligned}
& \gamma_{\alpha}:(0, \infty) \rightarrow \mathbb{Z}_{+} \text {rate of convergence of }\left(\alpha_{n}\right)_{n \in \mathbb{N}} \text { towards } 0, \\
& \gamma_{\beta}:(0, \infty) \rightarrow \mathbb{Z}_{+} \text {rate of convergence of }\left(\beta_{n}\right)_{n \in \mathbb{N}} \text { towards } 0, \\
& \psi_{\alpha}:(0, \infty) \rightarrow \mathbb{Z}_{+} \text {Cauchy modulus of }\left(\sum_{i=1}^{n}\left|\alpha_{i+1}-\alpha_{i}\right|\right), \\
& \psi_{\beta}:(0, \infty) \rightarrow \mathbb{Z}_{+} \text {Cauchy modulus of }\left(\sum_{i=1}^{n}\left|\beta_{i+1}-\beta_{i}\right|\right), \\
& \theta_{\beta}: \mathbb{Z}_{+} \rightarrow \mathbb{Z}_{+} \text {rate of divergence of }\left(\sum_{i=1}^{n} \beta_{i}\right) .
\end{aligned}
$$

Then for all $\varepsilon \in(0,2)$ and $g: \mathbb{N} \rightarrow \mathbb{N}$,

$$
\exists N \leq \Sigma\left(\varepsilon, g, M, \gamma_{\alpha}, \gamma_{\beta}, \psi_{\alpha}, \psi_{\beta}, \theta_{\beta}\right), \forall m, n \in[N ; N+g(N)] \quad\left(d\left(x_{n}, x_{m}\right) \leq \varepsilon\right),
$$

where

$$
\Sigma\left(\varepsilon, g, M, \gamma_{\alpha}, \gamma_{\beta}, \psi_{\alpha}, \psi_{\beta}, \theta_{\beta}\right):=\theta_{\beta}^{+}\left(\Gamma-1+\left\lceil\ln \left(\frac{12 M^{2}}{\varepsilon^{2}}\right)\right]\right)+1
$$


with $M \geq 4 \max \{d(u, p), d(x, p)\}$ for some $p \in \operatorname{Fix}(T)$,

$$
\begin{aligned}
& \tilde{\Phi}\left(\varepsilon, M, \theta_{\beta}, \psi_{\beta}, \psi_{\alpha}\right):=\theta_{\beta}\left(\max \left\{\psi_{\beta}\left(\frac{\varepsilon}{8 M}\right), \psi_{\alpha}\left(\frac{\varepsilon}{4 M}\right)\right\}+\ln \left\lceil\frac{M}{\varepsilon}\right\rceil+1\right)+1, \\
& \Phi\left(\varepsilon, M, \theta_{\beta}, \psi_{\beta}, \psi_{\alpha}, \gamma_{\alpha}, \gamma_{\beta}\right):=\max \left\{\tilde{\Phi}\left(\frac{\varepsilon}{2}, M, \theta_{\beta}, \psi_{\beta}, \psi_{\alpha}\right), \gamma_{\alpha}\left(\frac{\varepsilon}{4 M}\right), \gamma_{\beta}\left(\frac{\varepsilon}{4 M}\right)\right\} .
\end{aligned}
$$

The other constants are

$$
\begin{aligned}
& \varepsilon_{0}:=\frac{\varepsilon^{2}}{24(M+1)^{2}}, \quad \Gamma:=\max \left\{\chi_{k}^{*}\left(\varepsilon^{2} / 12\right):\left\lceil\frac{1}{\varepsilon_{0}}\right\rceil \leq k \leq \tilde{f}^{*\left(\left[M^{2} / \varepsilon_{0}^{2}\right\rceil\right)}(0)+\left\lceil\frac{1}{\varepsilon_{0}}\right\rceil\right\}, \\
& \chi_{k}^{*}(\varepsilon):=\tilde{\Phi}\left(\frac{\varepsilon}{4 M\left(\tilde{P}_{k}(\varepsilon / 2)+1\right)}\right)+\tilde{P}_{k}(\varepsilon / 2), \quad \tilde{P}_{k}(\varepsilon):=\left\lceil\frac{12 M^{2}(k+1)}{\varepsilon} \Phi\left(\frac{\varepsilon}{12 M(k+1)}\right)\right], \\
& \Delta_{k}^{*}(\varepsilon, g):=\frac{\varepsilon}{3 g_{\varepsilon, k}\left(\Theta_{k}(\varepsilon)-\chi_{k}^{*}(\varepsilon / 3)\right)}, \quad \Theta_{k}(\varepsilon):=\theta\left(\chi_{k}^{*}\left(\frac{\varepsilon}{3}\right)-1+\left\lceil\ln \left(\frac{3 M^{2}}{\varepsilon}\right)\right\rceil\right)+1, \\
& g_{\varepsilon, k}(n):=n+g\left(n+\chi_{k}^{*}\left(\frac{\varepsilon}{3}\right)\right), \quad \theta_{\beta}^{+}(n):=\max \left\{\theta_{\beta}(i): i \leq n\right\}, \\
& f(k):=\max \left\{\left\lceil\frac{M^{2}}{\Delta_{k}^{*}\left(\varepsilon^{2} / 4, g\right)}\right\rceil, k\right\}-k, \quad f^{*}(k)=f\left(k+\left\lceil\frac{1}{\varepsilon_{0}}\right\rceil\right)+\left\lceil\frac{1}{\varepsilon_{0}}\right\rceil, \\
& \tilde{f}^{*}(k):=k+f^{*}(k) .
\end{aligned}
$$

We now come to the metastability rates for the other set of conditions we consider for modified Halpern iterations.

Theorem 4.2 In the setting of Theorem 4.1, let $\left(\alpha_{n}\right)_{n \in \mathbb{N}} \subset[0,1]$ and $\left(\beta_{n}\right)_{n \in \mathbb{N}} \subset[0,1)$ satisfy (D1.a) and (D1.b), (D3.a) and (D3.b), (D4.a). Then the modified Halpern iteration $\left(x_{n}\right)_{n \in \mathbb{N}}$ is Cauchy. Furthermore, let

$$
\theta_{\beta}: \mathbb{Z}_{0} \rightarrow \mathbb{Z}_{+} \text {be a rate of convergence of }\left\{\prod_{n=1}^{N}\left(1-\beta_{n}\right)\right\} \text { towards } 0 .
$$

Then for all $\varepsilon \in(0,2)$ and $g: \mathbb{N} \rightarrow \mathbb{N}$,

$$
\exists N \leq \Sigma\left(\varepsilon, g, M, \gamma_{\alpha}, \gamma_{\beta}, \psi_{\alpha}, \psi_{\beta}, \theta_{\beta}\right), \forall m, n \in[N ; N+g(N)] \quad\left(d\left(x_{n}, x_{m}\right) \leq \varepsilon\right),
$$

where

$$
\Sigma\left(\varepsilon, g, M, \gamma_{\alpha}, \gamma_{\beta}, \psi_{\alpha}, \psi_{\beta}, \theta_{\beta}\right):=\max \left\{\Theta_{k}\left(\varepsilon^{2} / 4\right):\left\lceil\frac{1}{\varepsilon_{0}}\right\rceil \leq k \leq \tilde{f}^{\left(\left[\left\lceil M^{2} / \varepsilon_{0}^{2}\right\rceil\right)\right.}(0)+\left\lceil\frac{1}{\varepsilon_{0}}\right\rceil\right\},
$$

with $M \geq 4 \max \{d(u, p), d(x, p)\}$ for $p \in \operatorname{Fix}(T)$

$$
\begin{aligned}
& \tilde{\Phi}\left(\varepsilon, M, \theta_{\beta}, \psi_{\beta}, \psi_{\alpha}\right):=\theta_{\beta}\left(\frac{D \varepsilon}{M}\right)+1 \\
& \Phi\left(\varepsilon, M, \theta_{\beta}, \psi_{\beta}, \psi_{\alpha}, \gamma_{\alpha}, \gamma_{\beta}\right):=\max \left\{\tilde{\Phi}\left(\frac{\varepsilon}{2}, M, \theta_{\beta}, \psi_{\beta}, \psi_{\alpha}\right), \gamma_{\alpha}\left(\frac{\varepsilon}{4 M}\right), \gamma_{\beta}\left(\frac{\varepsilon}{4 M}\right)\right\},
\end{aligned}
$$




$$
\begin{aligned}
& 0<D \leq \prod_{n=1}^{\gamma(\varepsilon /(2 M)}\left(1-\beta_{n}\right), \\
& \gamma(\varepsilon):=\max \left\{\psi_{\alpha}\left(\frac{\varepsilon}{2 M}\right), \psi_{\beta}\left(\frac{\varepsilon}{4 M}\right)\right\}, \\
& \Theta_{k}(\varepsilon):=\theta\left(\frac{D_{k} \varepsilon}{3 M_{2}^{2}}\right)+1, \\
& 0<D_{k} \leq \prod_{n=1}^{\chi_{k}^{*}(\varepsilon / 3)-1}\left(1-\beta_{n}\right) .
\end{aligned}
$$

The other functionals and constants are defined as in Theorem 4.1.

\section{Remark 4.3}

1 The bounds in Theorems 4.1 and 4.2 only differ from the ones obtained in [1] for the usual Halpern iteration and the case of bounded $C$ by the new functionals $\Phi, \tilde{\Phi}$ which reflect the modification in the iteration scheme and by the fact that instead of $M \geq \operatorname{diam}(C)$ we only need $M \geq 4 \max \{d(u, p), d(x, p)\}$. Making only the latter change in the bounds in [1] yields rates of metastability for the usual Halpern iterations in the unbounded case.

2 The extractability of bounds on metastability depending only on the arguments shown can be explained in terms of general logical metatheorems from [14, 31]. In particular, the fact that $u, x, p$ and $C, X, T$ only enter these bounds via $M$ follows this way (note that we do not need any extra bound on $d(x, T(x))$ since $d(x, T(x)) \leq d(x, p)+d(p, T(p))+d(T(p), T(x)) \leq 2 d(x, p) \leq M)$. See [32] for details.

3 Again general logical metatheorems from [14,31] guarantee that the existence of a fixed point of $T$ can be relaxed to the existence of arbitrarily good approximate fixed points in some fixed $b$-bounded neighborhood around $x$, where then $M$ is taken to satisfy $M \geq 4(d(u, x)+b)+1$ (note that $d(u, p) \leq d(u, x)+d(x, p) \leq d(u, x)+b$ and that - reasoning as in the previous point - $d(x, T(x)) \leq 2 b+1 \leq M$ where, in fact, ' +1 ' can be replaced by an arbitrarily small positive number). See [32] for details.

4 The assumption of the CAT(0)-space $X$ to be complete and $C$ to be closed is actually not necessary as can be seen by going to the metric completion $\widehat{X}$ of $X$ and the closure $\bar{C}$ of $C$ in $\widehat{X}$, since $T$ extends to a nonexpansive operator on $\bar{C}[1$, Remark 4.5.(ii)].

\section{Estimates for modified Halpern iterations}

We need bounds for modified Halpern iterations. Part of the following result can be deduced from the proof of [3, Theorem 3.1].

Lemma 5.1 For modified Halpern iterations $\left(x_{n}\right)_{n \in \mathbb{N}}$ as in Definition 3.1, set $y_{n}:=\alpha_{n} x_{n} \oplus$ $\left(1-\alpha_{n}\right)$ Tx $x_{n}$ for all $n \in \mathbb{N}$. Then the following holds for $n \in \mathbb{Z}_{+}$in (1)-(3) and $n \in \mathbb{N}$ in (4)-(6):

(1) $d\left(x_{n+1}, x_{n}\right) \leq\left(1-\beta_{n}\right) d\left(y_{n}, y_{n-1}\right)+\left|\beta_{n}-\beta_{n-1}\right| d\left(u, y_{n-1}\right)$.

(2) $d\left(y_{n}, y_{n-1}\right) \leq \alpha_{n} d\left(x_{n}, x_{n-1}\right)+\left(1-\alpha_{n}\right) d\left(x_{n}, x_{n-1}\right)+\left|\alpha_{n}-\alpha_{n-1}\right| d\left(x_{n-1}, T x_{n-1}\right)$.

(3) $d\left(x_{n+1}, x_{n}\right) \leq\left(1-\beta_{n}\right) d\left(x_{n}, x_{n-1}\right)+\left(1-\beta_{n}\right)\left|\alpha_{n}-\alpha_{n-1}\right| d\left(x_{n-1}, T x_{n-1}\right)+$ $\left|\beta_{n}-\beta_{n-1}\right| \alpha_{n-1} d\left(x_{n-1}, T x_{n-1}\right)+\left|\beta_{n}-\beta_{n-1}\right| d\left(u, T x_{n-1}\right)$.

(4) $d\left(y_{n}, T x_{n}\right) \leq \alpha_{n} d\left(x_{n}, T x_{n}\right)$. 
(5) $d\left(x_{n+1}, T x_{n}\right) \leq \beta_{n} d\left(u, T x_{n}\right)+\left(1-\beta_{n}\right) \alpha_{n} d\left(x_{n}, T x_{n}\right)$.

(6) $d\left(x_{n}, T x_{n}\right) \leq d\left(x_{n}, x_{n+1}\right)+\beta_{n} d\left(u, T x_{n}\right)+\left(1-\beta_{n}\right) \alpha_{n} d\left(x_{n}, T x_{n}\right)$.

\section{Proof}

(1) Let $n \in \mathbb{Z}_{+}$.

$$
\begin{aligned}
d\left(x_{n+1}, x_{n}\right)= & d\left(\beta_{n} u \oplus\left(1-\beta_{n}\right) y_{n}, \beta_{n-1} u \oplus\left(1-\beta_{n-1}\right) y_{n-1}\right) \\
\leq & d\left(\beta_{n} u \oplus\left(1-\beta_{n}\right) y_{n}, \beta_{n} u \oplus\left(1-\beta_{n}\right) y_{n-1}\right) \\
& +d\left(\beta_{n} u \oplus\left(1-\beta_{n}\right) y_{n-1}, \beta_{n-1} u \oplus\left(1-\beta_{n-1}\right) y_{n-1}\right)
\end{aligned}
$$

by the triangle inequality

$$
\leq\left(1-\beta_{n}\right) d\left(y_{n}, y_{n-1}\right)+\beta_{n} d(u, u)+\left|\beta_{n}-\beta_{n-1}\right| d\left(u, y_{n-1}\right)
$$

by (W4) and (W2) from Definition 2.3 and 2.4

$$
\begin{aligned}
& =\left(1-\beta_{n}\right) d\left(y_{n}, y_{n-1}\right)+\left|\beta_{n}-\beta_{n-1}\right| d\left(u, y_{n-1}\right) \\
& =\left(1-\beta_{n}\right) d\left(y_{n}, y_{n-1}\right)+\left|\beta_{n}-\beta_{n-1}\right| d\left(u, \alpha_{n-1} x_{n-1} \oplus\left(1-\alpha_{n-1}\right) T x_{n-1}\right) .
\end{aligned}
$$

(2) Let $n \in \mathbb{Z}_{+}$.

$$
\begin{aligned}
d\left(y_{n}, y_{n-1}\right)= & d\left(\alpha_{n} x_{n} \oplus\left(1-\alpha_{n}\right) T x_{n}, \alpha_{n-1} x_{n-1} \oplus\left(1-\alpha_{n-1}\right) T x_{n-1}\right) \\
\leq & d\left(\alpha_{n} x_{n} \oplus\left(1-\alpha_{n}\right) T x_{n}, \alpha_{n} x_{n-1} \oplus\left(1-\alpha_{n}\right) T x_{n}\right) \\
& +d\left(\alpha_{n} x_{n-1} \oplus\left(1-\alpha_{n}\right) T x_{n}, \alpha_{n} x_{n-1} \oplus\left(1-\alpha_{n}\right) T x_{n-1}\right) \\
& +d\left(\alpha_{n} x_{n-1} \oplus\left(1-\alpha_{n}\right) T x_{n-1}, \alpha_{n-1} x_{n-1} \oplus\left(1-\alpha_{n-1}\right) T x_{n-1}\right)
\end{aligned}
$$

by the triangle inequality

$$
\begin{aligned}
\leq & \left(1-\alpha_{n}\right) \underbrace{d\left(T x_{n}, T x_{n}\right)}_{=0}+\alpha_{n} d\left(x_{n}, x_{n-1}\right)+\alpha_{n} \underbrace{d\left(x_{n-1}, x_{n-1}\right)}_{=0} \\
& +\left(1-\alpha_{n}\right) d\left(T x_{n}, T x_{n-1}\right)+\left|\alpha_{n}-\alpha_{n-1}\right| d\left(x_{n-1}, T x_{n-1}\right)
\end{aligned}
$$

by (W4) and (W2) from Definition 2.3 and 2.4

$$
\begin{aligned}
& =\alpha_{n} d\left(x_{n}, x_{n-1}\right)+\left(1-\alpha_{n}\right) d\left(T x_{n}, T x_{n-1}\right)+\left|\alpha_{n}-\alpha_{n-1}\right| d\left(x_{n-1}, T x_{n-1}\right) \\
& \leq \alpha_{n} d\left(x_{n}, x_{n-1}\right)+\left(1-\alpha_{n}\right) d\left(x_{n}, x_{n-1}\right)+\left|\alpha_{n}-\alpha_{n-1}\right| d\left(x_{n-1}, T x_{n-1}\right)
\end{aligned}
$$

since $T$ is nonexpansive.

(3) Let $n \in \mathbb{Z}_{+}$.

$$
\begin{aligned}
d\left(x_{n+1}, x_{n}\right) \leq & \left(1-\beta_{n}\right) d\left(y_{n}, y_{n-1}\right)+\left|\beta_{n}-\beta_{n-1}\right| d\left(u, \alpha_{n-1} x_{n-1} \oplus\left(1-\alpha_{n-1}\right) T x_{n-1}\right) \quad \text { by }(1) \\
\leq & \left(1-\beta_{n}\right)\left[\alpha_{n} d\left(x_{n}, x_{n-1}\right)+\left(1-\alpha_{n}\right) d\left(x_{n}, x_{n-1}\right)+\left|\alpha_{n}-\alpha_{n-1}\right| d\left(x_{n-1}, T x_{n-1}\right)\right] \\
& +\left|\beta_{n}-\beta_{n-1}\right| d\left(u, \alpha_{n-1} x_{n-1} \oplus\left(1-\alpha_{n-1}\right) T x_{n-1}\right) \quad \text { by }(2) \\
\leq & \left(1-\beta_{n}\right) d\left(x_{n}, x_{n-1}\right)+\left(1-\beta_{n}\right)\left|\alpha_{n}-\alpha_{n-1}\right| d\left(x_{n-1}, T x_{n-1}\right) \\
& +\left|\beta_{n}-\beta_{n-1}\right| \alpha_{n-1} d\left(u, x_{n-1}\right)+\left|\beta_{n}-\beta_{n-1}\right|\left(1-\alpha_{n-1}\right) d\left(u, T x_{n-1}\right)
\end{aligned}
$$

by Corollary 2.2 


$$
\begin{aligned}
\leq & \left(1-\beta_{n}\right) d\left(x_{n}, x_{n-1}\right)+\left(1-\beta_{n}\right)\left|\alpha_{n}-\alpha_{n-1}\right| d\left(x_{n-1}, T x_{n-1}\right) \\
& +\left|\beta_{n}-\beta_{n-1}\right| \alpha_{n-1}\left[d\left(u, x_{n-1}\right)-d\left(u, T x_{n-1}\right)\right] \\
& +\left|\beta_{n}-\beta_{n-1}\right| d\left(u, T x_{n-1}\right) \\
\leq & \left(1-\beta_{n}\right) d\left(x_{n}, x_{n-1}\right)+\left(1-\beta_{n}\right)\left|\alpha_{n}-\alpha_{n-1}\right| d\left(x_{n-1}, T x_{n-1}\right) \\
& +\left|\beta_{n}-\beta_{n-1}\right| \alpha_{n-1} d\left(x_{n-1}, T x_{n-1}\right)+\left|\beta_{n}-\beta_{n-1}\right| d\left(u, T x_{n-1}\right) \\
& \text { since } d\left(u, x_{n-1}\right) \leq d\left(u, T x_{n-1}\right)+d\left(T x_{n-1}, x_{n-1}\right) .
\end{aligned}
$$

(4) Let $n \in \mathbb{N}$. By Corollary 2.2,

$$
\begin{aligned}
d\left(y_{n}, T x_{n}\right) & =d\left(\alpha_{n} x_{n} \oplus\left(1-\alpha_{n}\right) T x_{n}, T x_{n}\right) \leq \alpha_{n} d\left(x_{n}, T x_{n}\right)+\left(1-\alpha_{n}\right) d\left(T x_{n}, T x_{n}\right) \\
& =\alpha_{n} d\left(x_{n}, T x_{n}\right) .
\end{aligned}
$$

(5) Let $n \in \mathbb{N}$. Again, by Corollary 2.2,

$$
\begin{aligned}
d\left(x_{n+1}, T x_{n}\right) & =d\left(\beta_{n} u \oplus\left(1-\beta_{n}\right) y_{n}, T x_{n}\right) \leq \beta_{n} d\left(u, T x_{n}\right)+\left(1-\beta_{n}\right) d\left(y_{n}, T x_{n}\right) \\
& \leq \beta_{n} d\left(u, T x_{n}\right)+\left(1-\beta_{n}\right) \alpha_{n} d\left(x_{n}, T x_{n}\right) \quad \text { by }(4) .
\end{aligned}
$$

(6) Let $n \in \mathbb{N}$. By the triangle inequality,

$$
\begin{aligned}
d\left(x_{n}, T x_{n}\right) & \leq d\left(x_{n}, x_{n+1}\right)+d\left(x_{n+1}, T x_{n}\right) \\
& \leq d\left(x_{n}, x_{n+1}\right)+\beta_{n} d\left(u, T x_{n}\right)+\left(1-\beta_{n}\right) \alpha_{n} d\left(x_{n}, T x_{n}\right) \quad \text { by (5). }
\end{aligned}
$$

For the general case of unbounded $C$, we also need some bound considerations, setting

$$
\begin{aligned}
& M_{0}:=\max \{d(x, p), d(u, p)\}, \\
& M_{1}:=2 d(p, u) \text { and } \\
& M_{2} \geq 4 M_{0}=4 \max \{d(u, p), d(x, p)\} \geq 2 M_{0}+M_{1}
\end{aligned}
$$

for a fixed point $p$ of $T$. Some parts of the next lemma are already implicit in the proof of $[4$, Theorem 1].

Lemma 5.2 Consider the modified Halpern iterations $\left(x_{n}\right)_{n \in \mathbb{N}}$. We define $y_{n}:=\alpha_{n} x_{n} \oplus(1-$ $\left.\alpha_{n}\right) T x_{n}$ for all $n \in \mathbb{N}$. Take $p \in \operatorname{Fix}(T)$. Then the following hold for $n \in \mathbb{N}$ :

(1) $d\left(y_{n}, p\right) \leq d\left(x_{n}, p\right)$.

(2) $d\left(x_{n+1}, p\right) \leq \beta_{n} d(u, p)+\left(1-\beta_{n}\right) d\left(x_{n}, p\right)$.

(3) $d\left(x_{n}, p\right) \leq M_{0}$. Hence $\left(x_{n}\right)_{n \in \mathbb{N}}$ and $\left(y_{n}\right)_{n \in \mathbb{N}}$ are bounded.

(4) $d\left(T x_{n}, p\right) \leq d\left(x_{n}, p\right) \leq M_{0}$.

(5) $d\left(x_{n}, T x_{n}\right) \leq 2 M_{0} \leq M_{2}$

(6) $d\left(x_{n}, u\right) \leq 2 M_{0} \leq M_{2}$.

(7) $d(u, T u) \leq 2 d(u, p)=M_{1} \leq M_{2}$.

(8) $d\left(T x_{n}, u\right) \leq M_{2}$.

(9) $d\left(x_{n+1}, x_{n}\right) \leq 2 M_{0} \leq M_{2}$. 
(1) Let $n \in \mathbb{N}$.

$$
\begin{aligned}
d\left(y_{n}, p\right) & =d\left(\alpha_{n} x_{n} \oplus\left(1-\alpha_{n}\right) T x_{n}, p\right), \\
& \leq \alpha_{n} d\left(x_{n}, p\right)+\left(1-\alpha_{n}\right) d\left(T x_{n}, p\right)
\end{aligned}
$$

by Corollary 2.2

$$
\begin{aligned}
& \leq \alpha_{n} d\left(x_{n}, p\right)+\left(1-\alpha_{n}\right) d\left(x_{n}, p\right) \quad \text { since } T \text { is nonexpansive } \\
& =d\left(x_{n}, p\right)
\end{aligned}
$$

(2) Let $n \in \mathbb{N}$.

$$
\begin{aligned}
d\left(x_{n+1}, p\right) & =d\left(\beta_{n} u \oplus\left(1-\beta_{n}\right) y_{n}, p\right) & \\
& \leq \beta_{n} d(u, p)+\left(1-\beta_{n}\right) d\left(y_{n}, p\right) & \text { by Corollary } 2.2 \\
& \leq \beta_{n} d(u, p)+\left(1-\beta_{n}\right) d\left(x_{n}, p\right) & \text { by }(1) .
\end{aligned}
$$

(3) The induction start is trivial. For $n \in \mathbb{N}$ the following holds:

$$
\begin{aligned}
d\left(x_{n+1}, p\right) & \leq \beta_{n} d(u, p)+\left(1-\beta_{n}\right) d\left(x_{n}, p\right) \text { by }(2) \\
& \leq \beta_{n} d(u, p)+\left(1-\beta_{n}\right) M_{0} \quad \text { by I.H., } \\
& \leq M_{0} .
\end{aligned}
$$

(4) This follows since $T$ is nonexpansive using (3).

(5) For $n \in \mathbb{N}, d\left(x_{n}, T x_{n}\right) \leq d\left(x_{n}, p\right)+d\left(p, T x_{n}\right) \leq 2 M_{0} \leq M_{2}$ using (3), (4).

(6) For $n \in \mathbb{N}, d\left(x_{n}, u\right) \leq d\left(x_{n}, p\right)+d(u, p) \leq 2 M_{0} \leq M_{2}$ using (3).

(7) $d(u, T u) \leq d(u, p)+d(p, T u) \leq 2 d(u, p)=M_{1} \leq M_{2}$.

(8) Let $n \in \mathbb{N}$.

$$
\begin{aligned}
d\left(T x_{n}, u\right) & \leq d\left(T x_{n}, T u\right)+d(T u, u) \leq d\left(x_{n}, u\right)+d(u, T u) \\
& \leq 2 M_{0}+M_{1} \leq M_{2}
\end{aligned}
$$

(9) For $n \in \mathbb{N}, d\left(x_{n+1}, x_{n}\right) \leq d\left(x_{n+1}, p\right)+d\left(x_{n}, p\right) \leq 2 M_{0} \leq M_{2}$

\section{Effective rates of asymptotic regularity}

In this section we give the actual quantitative convergence results. In the following let $(X, d)$ be a $\operatorname{CAT}(0)$ space. The results hold also true if we consider a W-hyperbolic space $(X, d, W)$. Let $C$ be a convex subset of $X$. Let $T: C \rightarrow C$ be nonexpansive.

The following proposition is the quantitative version of [3, Theorem 3.1].

Proposition 6.1 In the setting of Theorem 4.1, $\left(x_{n}\right)_{n \in \mathbb{N}}$ is an approximate fixed point sequence and $\lim _{n \rightarrow \infty} d\left(x_{n}, x_{n+1}\right)=0$. More precisely, for all $\varepsilon \in(0,2)$,

$$
\forall n \geq \tilde{\Phi}, \quad d\left(x_{n}, x_{n+1}\right) \leq \varepsilon \quad \text { and } \quad \forall n \geq \Phi, \quad d\left(x_{n}, T x_{n}\right) \leq \varepsilon,
$$


where

$$
\begin{aligned}
\tilde{\Phi} & :=\tilde{\Phi}\left(\varepsilon, M_{2}, \theta_{\beta}, \psi_{\beta}, \psi_{\alpha}\right):=\theta_{\beta}\left(\max \left\{\psi_{\beta}\left(\frac{\varepsilon}{8 M_{2}}\right), \psi_{\alpha}\left(\frac{\varepsilon}{4 M_{2}}\right)\right\}+\ln \left\lceil\frac{M_{2}}{\varepsilon}\right\rceil+1\right)+1 \\
\Phi & :=\Phi\left(\varepsilon, M_{2}, \theta_{\beta}, \psi_{\beta}, \psi_{\alpha}, \gamma_{\alpha}, \gamma_{\beta}\right) \\
& :=\max \left\{\tilde{\Phi}\left(\frac{\varepsilon}{2}, M_{2}, \theta_{\beta}, \psi_{\beta}, \psi_{\alpha}\right), \gamma_{\alpha}\left(\frac{\varepsilon}{4 M_{2}}\right), \gamma_{\beta}\left(\frac{\varepsilon}{4 M_{2}}\right)\right\}
\end{aligned}
$$

where $M_{2} \geq 4 \max \{d(u, p), d(x, p)\}$ for a $p \in \operatorname{Fix}(T)$.

Proof We want to apply [1, Lemma 5.5]. For all $n \in \mathbb{Z}_{+}$, we know

$d\left(u, T x_{n-1}\right) \leq M_{2} \quad$ by Lemma 5.2(8),

$d\left(x_{n-1}, T x_{n-1}\right) \leq 2 M_{0} \leq M_{2}$ by Lemma 5.2(5).

By Lemma 5.1(3), we have for all $n \in \mathbb{Z}_{+}$

$$
\begin{aligned}
d\left(x_{n+1}, x_{n}\right) \leq & \left(1-\beta_{n}\right) d\left(x_{n}, x_{n-1}\right)+\left(1-\beta_{n}\right)\left|\alpha_{n}-\alpha_{n-1}\right| d\left(x_{n-1}, T x_{n-1}\right) \\
& +\left|\beta_{n}-\beta_{n-1}\right| \alpha_{n-1} d\left(x_{n-1}, T x_{n-1}\right)+\left|\beta_{n}-\beta_{n-1}\right| d\left(u, T x_{n-1}\right) \\
\leq & \left(1-\beta_{n}\right) d\left(x_{n}, x_{n-1}\right)+\left|\alpha_{n}-\alpha_{n-1}\right| d\left(x_{n-1}, T x_{n-1}\right) \\
& +\left|\beta_{n}-\beta_{n-1}\right| d\left(x_{n-1}, T x_{n-1}\right)+\left|\beta_{n}-\beta_{n-1}\right| d\left(u, T x_{n-1}\right) \\
\leq & \left(1-\beta_{n}\right) d\left(x_{n}, x_{n-1}\right)+M_{2}\left[\left|\alpha_{n}-\alpha_{n-1}\right|+2\left|\beta_{n}-\beta_{n-1}\right|\right] .
\end{aligned}
$$

We set for all $n \in \mathbb{Z}_{+}$

$$
\begin{aligned}
& s_{n}:=d\left(x_{n}, x_{n-1}\right), \\
& a_{n}:=\beta_{n} \text { and } \\
& b_{n}:=M_{2}\left[\left|\alpha_{n}-\alpha_{n-1}\right|+2\left|\beta_{n}-\beta_{n-1}\right|\right] .
\end{aligned}
$$

Then for all $n \in \mathbb{Z}_{+}$

$$
\begin{aligned}
s_{n+1} & =d\left(x_{n+1}, x_{n}\right) \\
& \leq\left(1-\beta_{n}\right) d\left(x_{n}, x_{n-1}\right)+M_{2}\left[\left|\alpha_{n}-\alpha_{n-1}\right|+2\left|\beta_{n}-\beta_{n-1}\right|\right] \text { by (11) } \\
& =\left(1-a_{n}\right) s_{n}+b_{n} .
\end{aligned}
$$

The sequence $\left(s_{n}\right)$ is a priori bounded by $\frac{M_{2}}{2}$ by Lemma $5.2(9)$. But we know also by assumption that

$$
\begin{aligned}
& \sum_{n=1}^{\infty} \beta_{n} \rightarrow \infty, \quad \text { with the rate of divergence } \theta_{\beta}, \\
& \sum_{n=1}^{N} M_{2}\left(2\left|\beta_{n}-\beta_{n-1}\right|+\left|\alpha_{n}-\alpha_{n-1}\right|\right)<\infty, \quad N \rightarrow \infty, \text { with the Cauchy modulus, } \\
& \psi:(0, \infty) \rightarrow \mathbb{Z}_{+}, \quad \psi(\varepsilon)=\max \left\{\psi_{\beta}\left(\frac{\varepsilon}{4 M_{2}}\right), \psi_{\alpha}\left(\frac{\varepsilon}{2 M_{2}}\right)\right\} .
\end{aligned}
$$


We have fulfilled the requirements of [1, Lemma 5.5(i)]. Thus,

$$
\forall \varepsilon \in(0,2), \forall n \geq \tilde{\Phi} \quad s_{n} \leq \varepsilon
$$

where

$$
\begin{aligned}
& \tilde{\Phi}\left(\varepsilon, M_{2}, \theta_{\beta}, \psi_{\beta}, \psi_{\alpha}\right):= \\
& \quad \theta_{\beta}\left(\max \left\{\psi_{\beta}\left(\frac{\varepsilon}{8 M_{2}}\right), \psi_{\alpha}\left(\frac{\varepsilon}{4 M_{2}}\right)\right\}+\ln \left\lceil\frac{M_{2}}{\varepsilon}\right\rceil+1\right)+1 .
\end{aligned}
$$

It remains to determine $\Phi$. By Lemma 5.1(6), (9) and (10), we have

$$
\begin{aligned}
d\left(x_{n}, T x_{n}\right) & \leq d\left(x_{n}, x_{n+1}\right)+\beta_{n} d\left(u, T x_{n}\right)+\underbrace{\left(1-\beta_{n}\right.}_{\leq 1}) \alpha_{n} d\left(x_{n}, T x_{n}\right) \\
& \leq d\left(x_{n}, x_{n+1}\right)+\left(\beta_{n}+\alpha_{n}\right) M_{2} .
\end{aligned}
$$

We can define a rate of convergence

$$
\gamma:(0, \infty) \rightarrow \mathbb{Z}_{+}, \quad \text { with } \gamma(\varepsilon)=\max \left\{\gamma_{\alpha}\left(\frac{\varepsilon}{2 M_{2}}\right), \gamma_{\beta}\left(\frac{\varepsilon}{2 M_{2}}\right)\right\}
$$

such that the second term on the right becomes less than $\varepsilon$. For our bound, we then need to consider $\gamma\left(\frac{\varepsilon}{2}\right)$ so that the term on the right side becomes less than $\frac{\varepsilon}{2}$. In total, we get for all $\varepsilon \in(0,2)$ and for all $n \geq \Phi$, we have $d\left(x_{n}, T x_{n}\right) \leq \varepsilon$, where

$$
\begin{aligned}
& \tilde{\Phi}\left(\varepsilon, M_{2}, \theta_{\beta}, \psi_{\beta}, \psi_{\alpha}\right):=\theta_{\beta}\left(\max \left\{\psi_{\beta}\left(\frac{\varepsilon}{8 M_{2}}\right), \psi_{\alpha}\left(\frac{\varepsilon}{4 M_{2}}\right)\right\}+\ln \left\lceil\frac{M_{2}}{\varepsilon}\right\rceil+1\right)+1 \\
& \Phi\left(\varepsilon, M_{2}, \theta_{\beta}, \psi_{\beta}, \psi_{\alpha}, \gamma_{\alpha}, \gamma_{\beta}\right):=\max \left\{\tilde{\Phi}\left(\frac{\varepsilon}{2}, M_{2}, \theta_{\beta}, \psi_{\beta}, \psi_{\alpha}\right), \gamma_{\alpha}\left(\frac{\varepsilon}{4 M_{2}}\right), \gamma_{\beta}\left(\frac{\varepsilon}{4 M_{2}}\right)\right\} .
\end{aligned}
$$

We can also consider the case in which (D2.a) is replaced by (D4.a). This case was not considered by Cuntavepanit and Panyanak [3].

Proposition 6.2 In the setting of Theorem 4.2, $\left(x_{n}\right)_{n \in \mathbb{N}}$ is an approximate fixed point sequence and $\lim _{n \rightarrow \infty} d\left(x_{n}, x_{n+1}\right)=0$. More precisely, for all $\varepsilon \in(0,2)$,

$$
\forall n \geq \tilde{\Phi}, \quad d\left(x_{n}, x_{n+1}\right) \leq \varepsilon \quad \text { and } \quad \forall n \geq \Phi, \quad d\left(x_{n}, T x_{n}\right) \leq \varepsilon,
$$

where

$$
\begin{aligned}
\tilde{\Phi} & :=\tilde{\Phi}\left(\varepsilon, M_{2}, \theta_{\beta}, \psi_{\beta}, \psi_{\alpha}\right):=\theta_{\beta}\left(\frac{D \varepsilon}{M_{2}}\right)+1, \\
\Phi & :=\Phi\left(\varepsilon, M_{2}, \theta_{\beta}, \psi_{\beta}, \psi_{\alpha}, \gamma_{\alpha}, \gamma_{\beta}\right) \\
& :=\max \left\{\tilde{\Phi}\left(\frac{\varepsilon}{2}, M_{2}, \theta_{\beta}, \psi_{\beta}, \psi_{\alpha}\right), \gamma_{\alpha}\left(\frac{\varepsilon}{4 M_{2}}\right), \gamma_{\beta}\left(\frac{\varepsilon}{4 M_{2}}\right)\right\},
\end{aligned}
$$


where $M_{2}=4 \max \{d(u, p), d(x, p)\}$ for $p$ a fixed point of $T$ and

$$
\begin{aligned}
& \gamma(\varepsilon):=\max \left\{\psi_{\alpha}\left(\frac{\varepsilon}{2 M_{2}}\right), \psi_{\beta}\left(\frac{\varepsilon}{4 M_{2}}\right)\right\}, \\
& D \leq \prod_{n=1}^{\gamma(\varepsilon / 2)}\left(1-\beta_{n}\right) .
\end{aligned}
$$

Proof We want to use [1, Lemma 5.5(ii)]. We set again for $n \in \mathbb{Z}_{+}$,

$$
\begin{aligned}
& s_{n}:=d\left(x_{n}, x_{n-1}\right), \\
& a_{n}:=\beta_{n} \quad \text { and } \\
& b_{n}:=M_{2}\left[\left|\alpha_{n}-\alpha_{n-1}\right|+2\left|\beta_{n}-\beta_{n-1}\right|\right] .
\end{aligned}
$$

Then the main condition of [1, Lemma 5.5] is fulfilled by (12), since the sequences $\left(a_{n}\right)$, $\left(s_{n}\right)$ and $\left(b_{n}\right)$ were chosen the same.

The sequence $\left(s_{n}\right)$ is a priori bounded by $\frac{M_{2}}{2}$ by Lemma 5.2(9). But also,

$$
\begin{aligned}
& \prod_{n=1}^{\infty}\left(1-\beta_{n}\right)=0, \quad \text { with the rate of convergence } \theta_{\beta}, \\
& \sum_{n=1}^{N} M_{2}\left(2\left|\beta_{n}-\beta_{n-1}\right|+\left|\alpha_{n}-\alpha_{n-1}\right|\right)<\infty, \quad N \rightarrow \infty, \text { with the Cauchy modulus } \gamma,
\end{aligned}
$$

defined as $\gamma:(0, \infty) \rightarrow \mathbb{Z}_{+}$,

$$
\gamma(\varepsilon):=\max \left\{\psi_{\alpha}\left(\frac{\varepsilon}{2 M_{2}}\right), \psi_{\beta}\left(\frac{\varepsilon}{4 M_{2}}\right)\right\} .
$$

Then the conditions for Lemma [1, Lemma 5.5(ii)] are fulfilled, and we obtain the desired rate $\tilde{\Phi}$. The rate $\Phi$ is obtained as in Proposition 6.1.

\section{Quantitative properties of an approximate fixed point sequence}

Cuntavepanit and Panyanak's proof contains a lemma that uses the existence of Banach limits similar to the Banach limit lemma used in Saejung [7]. To make a current metatheorem applicable, this lemma has to be replaced in the proof. This can be done in the same way as carried out in [1].

Let $X$ be a complete CAT(0) space, $C \subset X$ be a closed convex subset and $T: C \rightarrow C$ be a nonexpansive mapping. For $t \in(0,1)$ and $u \in C$, consider

$$
T_{t}^{u}: C \rightarrow C, \quad T_{t}^{u} y=t u \oplus(1-t) T y
$$

One can easily see that $T_{t}^{u}$ is a strict contraction with a contractive constant $L=1-t$. Thus, $T_{t}^{u}$ has a unique fixed point $z_{t}^{u} \in C$ by Banach's fixed point theorem. Hence, $z_{t}^{u}$ solves the following equation uniquely:

$$
z_{t}^{u}=t u \oplus(1-t) T z_{t}^{u}
$$


The following proposition is our substitute for the use of Banach limits in convergence proofs of modified Halpern iterations.

Proposition 7.1 (See also [1, Proposition 9.1] for the bounded case) Let $\left(y_{n}\right)_{n \in \mathbb{N}}$ be a sequence in $C, u \in C, t \in(0,1)$, and let $z_{t}^{u}$ be as defined in (14). Define for all $n \in \mathbb{Z}_{+}$,

$$
\gamma_{n}^{t}:=(1-t) d\left(u, T z_{t}^{u}\right)^{2}-d\left(y_{n}, u\right)^{2} .
$$

Let $M \in \mathbb{Z}_{+}$be such that $d\left(z_{t}^{u}, y_{n}\right), d\left(y_{n}, T y_{n}\right), d\left(y_{n}, u\right) \leq M$ holds for all $n \in \mathbb{Z}_{+}$. Assume that $\left(y_{n}\right)_{n \in \mathbb{N}}$ is asymptotically regular and $\lim _{n \rightarrow \infty} d\left(y_{n}, y_{n+1}\right)=0$. Then

$$
\limsup _{n \rightarrow \infty} \gamma_{n}^{t} \leq 0
$$

Furthermore, if $\varphi$ is a rate of asymptotic regularity of $\left(y_{n}\right)_{n \in \mathbb{N}}$ and $\tilde{\varphi}$ is a rate of convergence of $\left(d\left(y_{n}, y_{n+1}\right)\right)_{n \in \mathbb{N}}$ towards 0 , then $\lim \sup _{n \rightarrow \infty} \gamma_{n}^{t} \leq 0$ with an effective rate $\psi$ defined by

$$
\psi(\varepsilon, t, M, \varphi, \tilde{\varphi})=\tilde{\varphi}\left(\frac{\varepsilon}{2 M(P(\varepsilon / 2, t, M, \varphi)+1)}\right)+P(\varepsilon / 2, t, M, \varphi),
$$

where

$$
P(\varepsilon, t, M, \varphi)=\left\lceil\frac{6 M^{2}}{t \varepsilon} \varphi\left(\frac{t \varepsilon}{6 M}\right)\right\rceil .
$$

Proof The result follows from the proof given in [1] by collecting all the instances of $M \geq$ $\operatorname{diam}(C)$ used in that proof.

Proposition 7.2 ([1, Proposition 9.3]) Let $\left(t_{k}\right)_{n \in \mathbb{N}} \subset(0,1)$ be non-increasing. For $k \in \mathbb{N}$ let $z_{t_{k}}^{u}$ be defined as in (14). Let the set $C$ be bounded with $\operatorname{diam}(C) \leq M \in \mathbb{N}$. Then for all $\varepsilon>0$ and $g: \mathbb{N} \rightarrow \mathbb{N}$, the following holds:

$$
\exists K_{0} \leq K(\varepsilon, g, M), \forall i, j \in\left[K_{0}, K_{0}+g\left(K_{0}\right)\right] \quad\left(d\left(z_{t_{i}}^{u}, z_{t_{j}}^{u}\right) \leq \varepsilon\right),
$$

where

$$
K(\varepsilon, g, M):=\tilde{g}^{\left\lceil M^{2} / \varepsilon^{2}\right\rceil}(0),
$$

and $\tilde{g}(k):=k+g(k)$.

Proof For the case of Hilbert spaces, the bound is extracted in [27] from Halpern's proof of the convergence of $\left(z_{t}^{u}\right)$. Since that proof extends unchanged to CAT(0) spaces, as remarked by Kirk [33], the same is true for the extracted bound.

Lemma 7.3 (Compare Saejung [7, Lemma 2.2]) Let $z_{t}^{u}$ be defined as in Eq. (14). If $\operatorname{Fix}(T) \neq$ $\emptyset$, then

$$
d\left(p, z_{t}^{u}\right) \leq d(p, u), \quad \text { for } p \in \operatorname{Fix}(T) .
$$

In particular, $d\left(u, z_{t}^{u}\right), d\left(x_{n}, z_{t}^{u}\right), d\left(u, T z_{t}^{u}\right) \leq M_{2}$ for all $n \in \mathbb{N}$. 
Proof Let $p \in \operatorname{Fix}(T)$. Then

$$
\begin{aligned}
d\left(p, z_{t}^{u}\right) & =d\left(p, t u \oplus(1-t) T z_{t}^{u}\right) \leq t d(p, u)+(1-t) d\left(p, T z_{t}^{u}\right) \quad \text { by Corollary } 2.2 \\
& \leq t d(p, u)+(1-t) d\left(p, z_{t}^{u}\right) .
\end{aligned}
$$

Hence, $d\left(p, z_{t}^{u}\right) \leq d(p, u)$.

Then, by the definition of $M_{0}$ in (6) and by Lemma 5.2(3),

$$
\begin{aligned}
& d\left(z_{t}^{u}, u\right) \leq d\left(z_{t}^{u}, p\right)+d(p, u) \leq 2 d(p, u) \leq M_{2}, \\
& d\left(z_{t}^{u}, x_{n}\right) \leq d\left(z_{t}^{u}, p\right)+d\left(p, x_{n}\right) \leq d(p, u)+M_{0} \leq M_{2}, \\
& d\left(T z_{t}^{u}, u\right) \leq d\left(T z_{t}^{u}, p\right)+d(u, p) \leq d\left(z_{t}^{u}, p\right)+d(u, p) \leq M_{2}
\end{aligned}
$$

for all $n \in \mathbb{N}$.

With this result, we can generalize Proposition 7.2 to unbounded domains given a fixed point $p$ of $T$.

Corollary 7.4 In the situation of Proposition 7.2, the conclusion also holds if $C$ is unbounded and $T$ has a nonempty fixed point set. In this case, the bound $M$ can be replaced by $M_{2}$.

Proof By the logical analysis of Halpern's proof [18, Theorem 1] in [27, Theorem 4.2], one can replace the bound $M$ on the diameter by a bound on $d\left(z_{t}^{u}, u\right)$. If we have a fixed point $p$ of $T$ at our disposal, we can take this bound to be $M_{2} \geq 4 \max \{d(u, p), d(x, p)\}$ by Lemma 7.3.

The next lemma for modified Halpern iterations interestingly is precisely of the form proved for the usual Halpern iterations (for bounded $C$ ) in [1, Lemma 9.2] though the proof is different.

Lemma 7.5 Let $u, x \in C$ and $\left(x_{n}\right)_{n \in \mathbb{N}}$ be the modified Halpern iterations as in Definition 3.1. Then for all $t \in(0,1)$ and $n \geq 0$,

$$
d\left(x_{n+1}, z_{t}^{u}\right)^{2} \leq\left(1-\beta_{n}\right) d\left(x_{n}, z_{t}^{u}\right)^{2}+\beta_{n}\left((1-t) d\left(u, T z_{t}^{u}\right)^{2}-d\left(x_{n+1}, u\right)^{2}\right)+M_{2}^{2} t,
$$

with $M_{2}:=4 \max \{d(u, p), d(x, p)\}$.

Proof Let $n \in \mathbb{N}$ and $t \in(0,1)$ be given.

We need the following inequalities which follow from (2):

$$
\begin{aligned}
& d\left(x_{n+1}, u\right)^{2}=d\left(\beta_{n} u \oplus\left(1-\beta_{n}\right) y_{n}, u\right)^{2} \leq\left(1-\beta_{n}\right) d\left(y_{n}, u\right)^{2}, \\
& \begin{aligned}
d\left(u, z_{t}^{u}\right)^{2}= & d\left(u, t u \oplus(1-t) T z_{t}^{u}\right)^{2} \leq(1-t) d\left(T z_{t}^{u}, u\right)^{2}, \\
d\left(x_{n+1}, z_{t}^{u}\right)^{2} & =d\left(\beta_{n} u \oplus\left(1-\beta_{n}\right) y_{n}, z_{t}^{u}\right)^{2} \\
& \leq\left(1-\beta_{n}\right) d\left(y_{n}, z_{t}^{u}\right)^{2}+\beta_{n} d\left(u, z_{t}^{u}\right)^{2}-\beta_{n} \underbrace{\left.1-\beta_{n}\right) d\left(u, y_{n}\right)^{2}}_{\geq d\left(x_{n+1}, u\right)^{2}} \text { by (2) }
\end{aligned}
\end{aligned}
$$


in the next step, we apply (2) on $d\left(y_{n}, z_{t}^{u}\right)^{2}$ and use again (17)

$$
\begin{aligned}
& \leq\left(1-\beta_{n}\right)[\alpha_{n} d\left(x_{n}, z_{t}^{u}\right)^{2}+\left(1-\alpha_{n}\right) d\left(T x_{n}, z_{t}^{u}\right)^{2} \underbrace{-\alpha_{n}\left(1-\alpha_{n}\right) d\left(x_{n}, T x_{n}\right)^{2}}_{\leq 0}] \\
& +\beta_{n} d\left(u, z_{t}^{u}\right)^{2}-\beta_{n} d\left(x_{n+1}, u\right)^{2} \\
& \leq\left(1-\beta_{n}\right) \alpha_{n} d\left(x_{n}, z_{t}^{u}\right)^{2}+\left(1-\beta_{n}\right)\left(1-\alpha_{n}\right) d\left(T x_{n}, z_{t}^{u}\right)^{2} \\
& +\beta_{n} d\left(u, z_{t}^{u}\right)^{2}-\beta_{n} d\left(x_{n+1}, u\right)^{2} \\
& \leq\left(1-\beta_{n}\right) \alpha_{n} d\left(x_{n}, z_{t}^{u}\right)^{2} \\
& +\underbrace{\left(1-\beta_{n}\right)\left(1-\alpha_{n}\right)}_{\leq 1}\left[t d\left(u, T x_{n}\right)^{2}\right. \\
& +\underbrace{(1-t)}_{\leq 1} \underbrace{d\left(T z_{t}^{u}, T x_{n}\right)^{2}}_{\leq d\left(z_{t}^{u}, x_{n}\right)^{2}} \underbrace{\left.-t(1-t) d\left(u, T z_{t}^{u}\right)^{2}\right]}_{\leq 0} \text { by (2) } \\
& +\beta_{n} d\left(u, z_{t}^{u}\right)^{2}-\beta_{n} d\left(x_{n+1}, u\right)^{2} \\
& \leq\left(1-\beta_{n}\right) \alpha_{n} d\left(x_{n}, z_{t}^{u}\right)^{2}+\left(1-\beta_{n}\right)\left(1-\alpha_{n}\right) d\left(x_{n}, z_{t}^{u}\right)^{2}+\beta_{n} \underbrace{d\left(u, z_{t}^{u}\right)^{2}}_{\leq(1-t) d\left(T z_{t}^{u}, u\right)^{2}} \\
& -\beta_{n} d\left(x_{n+1}, u\right)^{2}+t d\left(u, T x_{n}\right)^{2} \\
& \leq\left(1-\beta_{n}\right) d\left(x_{n}, z_{t}^{u}\right)^{2}+\beta_{n}\left[(1-t) d\left(T z_{t}^{u}, u\right)^{2}-d\left(x_{n+1}, u\right)^{2}\right] \\
& +t d\left(u, T x_{n}\right)^{2} \text { by (18) } \\
& \leq\left(1-\beta_{n}\right) d\left(x_{n}, z_{t}^{u}\right)^{2}+\beta_{n}\left[(1-t) d\left(T z_{t}^{u}, u\right)^{2}-d\left(x_{n+1}, u\right)^{2}\right] \\
& +t M_{2}^{2} \text { by } 5.2(8) \text {. }
\end{aligned}
$$

\section{Proof of Theorems 4.1 and 4.2 concluded}

The proof of Theorem 4.1 (and also of Theorem 4.2) is essentially the same as the proof of the existence of a rate of metastability for ordinary Halpern iterations in the case of bounded $C$ by Kohlenbach and Leuştean [1, Theorem 4.2] replacing the rates of asymptotic regularity $\Phi, \tilde{\Phi}$ used in [1] by the new ones we obtained in Section 6 . This is due to the fact that despite the different iteration scheme at hand, Lemma 7.5 (though by a different proof) is identical to [1, Lemma 9.2]. The only other thing we have to check is that we can use the bound $M:=M_{2} \geq 4 \max \{d(u, p), d(x, p)\}$ in Proposition 7.1. This, however, follows from Lemma 7.3 and Lemma 5.2(5) and (6).

\section{Competing interests}

The authors declare that they have no competing interests.

\section{Authors' contributions}

This paper is based on parts of the master thesis of the first author written under the supervision of the second author. All authors read and approved the final manuscript.

\section{Acknowledgements}

The 2 nd author has been supported by the German Science Foundation (DFG Project KO 1737/5-1).

Received: 25 June 2012 Accepted: 10 October 2012 Published: 29 October 2012

\section{References}

1. Kohlenbach, U, Leuştean, L: Effective metastability of Halpern iterates in CAT(0) spaces. Adv. Math. 231, 2525-2556

(2012) 
2. Kohlenbach, U, Leuştean, L: On the computational content of convergence proofs via Banach limits. Philos. Trans. R. Soc. A, Math. Phys. Eng. Sci. 370, 3449-3463 (2012)

3. Cuntavepanit, A, Panyanak, B: Strong convergence of modified Halpern iterations in CAT(0) spaces. Fixed Point Theory Appl. 2011, 869458 (2011)

4. Kim, T-H, Xu, H-K: Strong convergence of modified Mann iterations. Nonlinear Anal. 61, 51-60 (2005)

5. Tao, T: Soft analysis, hard analysis, and the finite convergence principle. Blog entry. http://terrytao.wordpress.com/ 2007/05/23/soft-analysis-hard-analysis-and-the-finite-convergence-principle/. Acessed 20 April 2010. Also published 2009 in [15]

6. Kohlenbach, U: Applied Proof Theory. Proof Interpretations and Their Use in Mathematics. Springer Monographs in Mathematics. Springer, Berlin (2008)

7. Saejung, S: Halpern's iteration in CAT(0) spaces. Fixed Point Theory Appl. 2010, Article ID 471781 (2010)

8. Avigad, J, Gerhardy, P, Towsner, H: Local stability of ergodic averages. Trans. Am. Math. Soc. 362, 261-288 (2010)

9. Gromov, M: Hyperbolic groups. In: Gersten, S (ed.) Essays in Group Theory, pp. 75-263. Springer, New York (1987)

10. Bridson, MR, Haefliger, A: Metric Spaces of Non-Positive Curvature. Grundlehren der Mathematischen Wissenschaften. Springer, Berlin (2010). Softcover reprint of hardcover 1st ed. 1999

11. Dhompongsa, S, Panyanak, B: On $\Delta$-convergence theorems in CAT(0) spaces. Comput. Math. Appl. 56, 2572-2579 (2008)

12. Takahashi, W: A convexity in metric space and nonexpansive mappings. Kodai Math. Semin. Rep. 22, 142-149 (1970)

13. Goebel, K, Reich, S: Uniform Convexity, Hyperbolic Geometry, and Nonexpansive Mappings. Monographs and Textbooks in Pure and Applied Mathematics. Dekker, New York (1984)

14. Kohlenbach, U: Some logical metatheorems with applications in functional analysis. Trans. Am. Math. Soc. 357, 89-128 (2005)

15. Tao, T: Structure and Randomness: Pages from Year One of a Mathematical Blog. Am. Math. Soc., Boston (2009)

16. Kreisel, G: On the interpretation of non-finitist proofs. Part 1. J. Symb. Log. 16, 241-267 (1951)

17. Kreisel, G: On the interpretation of non-finitist proofs. Part 2. J. Symb. Log. 17, 43-58 (1952)

18. Halpern, B: Fixed points of nonexpanding maps. Bull. Am. Math. Soc. 73, 957-961 (1967)

19. Lions, P-L: Approximation de points fixes de contractions. C. R. Acad. Sci. Ser. A 284, A1357-A1359 (1977)

20. Reich, S: Some problems and results in fixed point theory. In: Singh, S, Thomeier, S, Watson, B (eds.) Contemporary Mathematics. Topological Methods in Nonlinear Functional Analysis, vol. 21, pp. 179-187 (1983)

21. Wittmann, R: Approximation of fixed points of nonexpansive mappings. Arch. Math. (Basel) 58, 486-491 (1992)

22. Reich, S: Strong convergence theorems for resolvents of accretive operators in Banach spaces. J. Math. Anal. Appl. 75 , 287-292 (1980)

23. Reich, S: Approximating fixed points of nonexpansive mappings. Panam. Math. J. 4, $23-28$ (1994)

24. Shioji, N, Takahashi, W: Strong convergence of approximated sequences for nonexpansive mappings in Banach spaces. Proc. Am. Math. Soc. 125, 3641-3645 (1997)

25. Kopecka, E, Reich, S: A note on the approximation of fixed points in the Hilbert ball. J. Nonlinear Convex Anal. 9, 361-367 (2008)

26. Piątek, B: Halpern iteration in CAT( $\kappa)$ spaces. Acta Math. Sin. Engl. Ser. 27, 635-646 (2011)

27. Kohlenbach, U: On quantitative versions of theorems due to F.E. Browder and R. Wittmann. Adv. Math. 226, 2764-2795 (2011)

28. Leuştean, L: Rates of asymptotic regularity for Halpern iterations of nonexpansive mappings. J. Univers. Comput. Sci. 13, 1680-1691 (2007)

29. Suzuki, T: A sufficient and necessary condition for Halpern-type strong convergence to fixed points of nonexpansive mappings. Proc. Am. Math. Soc. 135, 99-106 (2007)

30. Chidume, CE, Chidume, CO: Iterative approximation of fixed points of nonexpansive mappings. J. Math. Anal. Appl. 318, 288-295 (2006)

31. Gerhardy, P, Kohlenbach, U: General logical metatheorems for functional analysis. Trans. Am. Math. Soc. 360, 2615-2660 (2008)

32. Schade, KC: Proof mining for Halpern iterations in CAT(0) spaces. Master thesis, TU, Darmstadt (2012)

33. Kirk, W: Geodesic geometry and fixed point theory. In: Seminar of Mathematical Analysis (Malaga, Seville, 2002-2003), vol. 64, pp. 195-225. University of Sevilla Secretary, Sevilla (2003)

doi:10.1186/1687-1812-2012-191

Cite this article as: Schade and Kohlenbach: Effective metastability for modified Halpern iterations in CAT(0) spaces.

Fixed Point Theory and Applications 2012 2012:191. 